\title{
Valor pronóstico de los nódulos tumorales extranodales en el mesorrecto en pacientes operados por cáncer de recto*

\author{
Mesorectal extranodal deposits. Impact on survival
}

\author{
Drs. M.E. MOLINA P. ${ }^{1}$, J.M. ZÚÑIGA A. ${ }^{1}$, G. TAPIA N. ${ }^{1}$, A. ZÁRATE C. ${ }^{1}$, \\ I. DUARTE DE C. ${ }^{2}$, J. TORRES M. ${ }^{2}, A$. ZÚNIGA D. ${ }^{1}$ \\ ${ }^{1}$ Departamento de Cirugía Digestiva. ${ }^{2}$ Departamento de Anatomía Patológica. Facultad de Medicina. \\ Pontificia Universidad Católica de Chile. Santiago, Chile
}

\begin{abstract}
RESUMEN
Depósitos o nódulos tumorales extranodales (NTM) en el mesorrecto, y discontinuos con el tumor primario se demuestran con frecuencia en el estudio anatomopatológico de la pieza quirúrgica de pacientes sometidos a resección radical por adenocarcinoma del recto. El conocimiento actual sugiere que la presencia de estos nódulos sería un indicador de mal pronóstico. El objetivo de esta comunicación es estudiar el impacto que tiene la presencia de estos NTM en el pronóstico de los pacientes sometidos a resección radical. Material y método. Retrospectivamente se revisaron los cortes histológicos de 250 pacientes operados entre los años 1985 - 2003. Las características anatomopatológicas y la evolución de aquellos pacientes en los que se demostraron NTM fue comparado con un grupo sin estos nódulos, seleccionado al azar. Se consignaron datos demográficos, clínicos y anatomopatológicos. Para el análisis de sobrevida se excluyeron los pacientes en etapa IV y aquellos sometidos a resección paliativa. El análisis estadístico se realizó con Test de Anova y Chi2. Se consideró significativo $p<0.05$. para el análisis de sobrevida actuarial se utilizó el método de Kaplan Meier. Resultados: NTM fueron demostrados en 41 de 250 pacientes (16\%). Entre los 209 restantes se seleccionó aleatoriamente 65 pacientes sin NTM como grupo control. Ambos grupos son comparables para edad, sexo, etapa de enfermedad y diferenciación tumoral. Así, la serie está compuesta por 106 pacientes: 41 con NTM y 65 sin NTM. Edad promedio: 64.5 años (i: 30-88). Hombres 53\%. De acuerdo al estudio anatomopatológico 60 pacientes (57\%) se encontraban en etapas I y II; $33(31 \%)$ en etapa III y 13 (12\%) en etapa IV. El análisis estadístico demostró que tumores pobremente diferenciados, presencia de invasión linfática y perineural, metástasis en los ganglios linfáticos y etapa avanzada de la enfermedad tenían una asociación estadísticamente significativa con la presencia de NTM. Sin embargo, en el seguimiento no hubo una diferencia estadísticamente significativamente en la sobrevida actuarial a 5 y 10 años en pacientes con y sin NTM. Se concluye que la presencia de NTM se relaciona con una etapa avanzada de la enfermedad y que su impacto en la sobrevida y recurrencia local necesita ser analizada prospectivamente en una serie más extensa.
\end{abstract}

PALABRAS CLAVE: Nódulos extranodales, cáncer de recto, pronóstico.

*Recibido el 9 de Enero de 2006 y aceptado para publicación el 18 de Abril de 2006

Correspondencia: Dr. M.E. Molina

Marcoleta 347, Santiago, Chile

e mail: memolina@puc.cl 


\section{SUMMARY}

Mesorectal extranodal deposits, discontinuous with the primary tumors, are identified in the specimen of a significant number of rectal adenocarcinomas of patients submitted to radical resection. Current knowledge tends to consider these as indicators of poor prognosis. The aim of this study is to analyze the impact of these deposits in the outcome of patients whit rectal cancer. Retrospectively the histology slides of 250 patients operated between years 1985-2003 were reviewed. The pathological characteristics and outcome of those in which deposits were demonstrated were compared with a randomly selected group without them. Tumoral deposits were demonstrated in 41 of the $250(16 \%)$ cases. Sixty-five patients of the same cohort, without deposits, were randomly selected as a control group. Both groups are comparable for age, sex, stage of the disease and tumor differentiation. Therefore the study population comprise 106 patients: 41 with extranodal deposits and 65 without them. Age x: 64.5 (i:30-88), men: $53 \%$. According to the pathology report 60 patients (57\%) were stage I and II; $33(31 \%)$ stage III and $13(12 \%)$ stage IV. The statistical analysis showed that poorly differentiated tumors, lymphatic and perineural invasion, lymph node metastasis and advanced stage had a statistically significant association with the presence of tumor deposits in the mesorectum. However, at follow-up there was no significant statistical difference in 5 and 10 year actuarial survival in patients with and without tumor deposits. It is concluded that these mesorectal extranodal deposits are related to an advanced stage of the disease and its impact on survival and local recurrence needs to be analyzed in a larger series.

\section{KEY WORDS: Mesorectal extranodal deposits, rectal cancer, impact on survival.}

\section{INTRODUCCIÓN}

La penetración del tumor en la pared rectal y la presencia de metástasis linfáticas en el mesorrecto son dos reconocidos e importantes factores pronósticos para la sobrevida y recidiva local después de la resección del cáncer del recto. La invasión vascular y perineural son otros dos factores pronósticos. Reconocer estos hechos ha demandado a los patólogos un sistemático y acucioso estudio de las piezas operatorias con el afán de poder precisar el pronóstico del paciente sometido a una resección curativa. Esta metódica ha permitido identificar en el mesorrecto de las piezas operatorias la presencia de nódulos o depósitos tumorales extranodales (NTM) que se encuentran en el mesorrecto en discontinuidad con el tumor primario y con los ganglios linfáticos y que no son detectables en el estudio preoperatorio ni en la exploración quirúrgica. Pocos estudios han sido publicados en relación a los NTM y su importancia pronóstica. Fueron reconocidos por el American Joint Committee on Cancer en su manual para etapificación del cáncer publicado en 19981. En esta publicación se considera a los NTM como metástasis linfáticas regionales (N1). El Royal College of Pathologists en el Reino Unido establece que "depósitos tumorales extramurales que miden 3 ó más mms se consideran como ganglios linfáticos comprometidos aunque no se reconozca estructura linfática residual. Depósitos tumorales menores serían aparentemente extensión discontinua del tumor primario"2. Este límite arbitrario en el tamaño del NTM obedecería a que raramente un ganglio linfático con metástasis tumoral mide menos de $3 \mathrm{~mm}$. Así entonces, se sugiere que algunos de estos nódulos serían ganglios linfáticos reemplazados totalmente por tumor y otros, particularmente los menores de $3 \mathrm{mms}$ o microscópicos, serían extensión discontinua del tumor primitivo o metástasis por vía posiblemente hematógena. Cualquiera que sea su patogenia es importante conocer su efecto sobre el pronóstico de la enfermedad. El objeto de este estudio es conocer las características anatomopatológicas de estas lesiones y su posible impacto pronóstico sobre la recidiva local y sobrevida en nuestra serie de pacientes sometidos a resección del recto por adenocarcinoma.

\section{MATERIAL Y MÉTODO}

En este estudio retrospectivo se revisaron, en el Departamento de Anatomía Patológica, los cortes histopatológicos de las piezas operatorias de 250 pacientes operados por cáncer de recto entre los años 1985 y 2003. Para el estudio anatomopatológico de las piezas operatorias, el recto y el mesorrecto fueron fijados en formalina y luego seccionados en cortes transversales para su observación macro y microscópica de acuerdo a la técnica descrita por Scott y Quirke ${ }^{3}$ (Figura 1). Se seleccionó todos aquellos pacientes en los cuales el estudio histopatológico de la pieza operatoria demostró la presencia de Nódulos Tumorales en el 


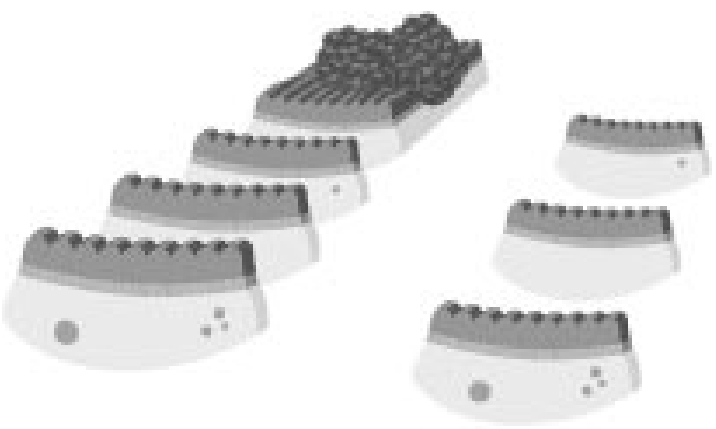

Figura 1. Técnica de Scout y Quirke para el estudio anatomopatológico del mesorrecto y recto.

Mesorrecto (NTM). Entre los pacientes restantes se eligió al azar un grupo de ellos sin NTM, como un grupo control.

Se revisaron los registros clínicos y anatomopatológicos, para el análisis de factores que se asocien con la presencia de NTM. Para la etapificación de los tumores no se consideró la presencia de NTM. Además, se analizó la sobrevida comparando ambos grupos según datos del seguimiento obtenidos de la revisión de los registros clínicos, entrevistas personales o telefónicas y datos de defunciones del Registro Civil. Para el análisis de la sobrevida se excluyeron los pacientes en etapa IV al momento de la cirugía y aquellos sometidos a resección paliativa.

El análisis estadístico se realizó con test de regresión logística (ANOVA) y Chi Cuadrado. Se consideró significativo un $\mathrm{n}<0.05$. Para el análisis de sobrevida actuarial se utilizó el método de Kaplan Meier.

\section{RESULTADOS}

Se demostraron NTM en 41 de las 250 (16\%) piezas operatorias de los pacientes operados por cáncer del recto en el período mencionado (Figura 2). Entre los 209 restantes se seleccionaron al azar un grupo de 65 pacientes como control. Ambos grupos eran comparables en edad, sexo, etapa de su enfermedad y diferenciación tumoral. Así, la muestra está constituída por un total de 106 pacientes: 41 con NTM y 65 sin NTM. Hombres 53\%, mujeres $47 \%$. Edad promedio de 64.5 años (i: $30-$ 88). Se practicó una resección anterior baja o ultrabaja en 86 pacientes; una resección abdominoperineal en 16 y una operación de Hartmann en 4. Se realizó resección paliativa en 6 pacientes $(5,6 \%)$. El estudio histopatológico de las piezas operatorias demostró que 60 pacientes (57\%) se

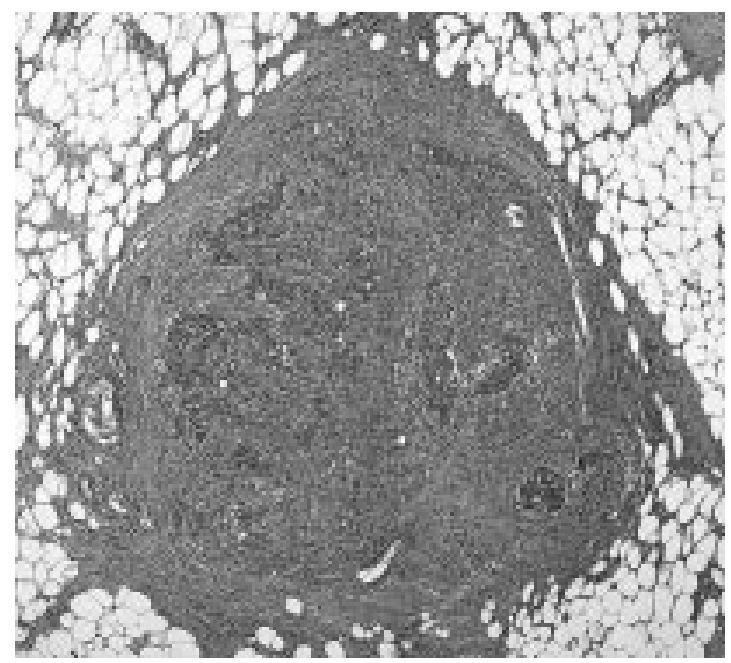

Figura 2. Metástasis nodular extranodal en el mesorrecto de un paciente operado por cáncer de recto.

encontraban en etapa I - II, 33 (31\%) en etapa III y $13(12 \%)$ en etapa IV.

En la Tabla 1 se analiza la presencia o ausencia de NTM, según la administración preoperatorio de XRT-QMT, grado de diferenciación tumoral, presencia de permeaciones linfáticas, vasculares o perineurales y etapa tumoral. Al realizar el análisis estadístico se encontró que el menor grado de diferenciación, la presencia de permeaciones linfáticas, perineurales, las metástasis ganglionares y la etapa tumoral más avanzada se relacionan en forma estadísticamente significativa con la presencia de NTM. No se relaciona de manera estadística significativa con la presencia de NTM, la administración de XRT-QMT preoperatorio, la profundidad del tumor en la pared rectal, y el hallazgo de un componente mucinoso o de permeaciones vasculares.

Se obtuvo un seguimiento del $100 \%$ de los pacientes. Hubo recurrencia en 49 pacientes: 20 con NTM y 29 sin NTM. Al momento de cerrar el estudio 47 pacientes habían fallecido: 19 con NTM y 28 sin NTM. La sobrevida actuarial a 5 y 10 años fue de $54 \%$ y $36 \%$ respectivamente para los pacientes con NTM y de $60 \%$ y $59 \%$ respectivamente para los pacientes sin NTM ( $p$ : NS). Al realizar el análisis de sobrevida y recurrencia de la enfermedad no se demostró diferencia estadísticamente significativa entre los grupos con y sin NTM.

\section{DISCUSIÓN}

Algunos pacientes con cáncer del recto a pesar de haber sido sometidos a una resección total 
Tabla 1

PRESENCIA O AUSENCIA DE NTM EN PACIENTES OPERADOS POR CÁNCER DE RECTO

\begin{tabular}{|c|c|c|c|c|c|c|}
\hline \multirow[b]{2}{*}{ XRT-QMT preoperatorio } & \multicolumn{2}{|c|}{$\begin{array}{c}\text { Con NTM (\%) } \\
T=41\end{array}$} & \multicolumn{2}{|c|}{$\begin{array}{c}\operatorname{Sin} N T M(\%) \\
T=65\end{array}$} & \multirow{2}{*}{$\begin{array}{r}\text { Total } \\
31\end{array}$} & \multirow{2}{*}{$\begin{array}{c}p \\
\text { NS }\end{array}$} \\
\hline & 12 & $(29,2)$ & 19 & $(29,3)$ & & \\
\hline Bien diferenciado & 1 & $(2,4)$ & 20 & $(30,7)$ & 21 & \\
\hline Mod. diferenciado & 32 & (78) & 35 & $(53,8)$ & 67 & 0,02 \\
\hline Indiferenciado & 8 & $(19,5)$ & 10 & $(15,4)$ & 18 & \\
\hline Perm. linfáticas & 26 & $(63,4)$ & 13 & $(20)$ & 39 & $<0,01$ \\
\hline Perm. vasculares & 7 & $(17)$ & 5 & $(7,7)$ & 12 & NS \\
\hline Perm. perineurales & 7 & $(17)$ & 2 & $(3,0)$ & 9 & 0,01 \\
\hline Mucinoso & 5 & $(12,2)$ & 10 & $(15,4)$ & 15 & NS \\
\hline Ganglios (+) & 26 & $(63,4)$ & 14 & $(21,5)$ & 40 & $<0,01$ \\
\hline Ganglios (-) & 15 & $(36,6)$ & 51 & $(78,5)$ & 66 & $<0,01$ \\
\hline Etapa I & 2 & $(4,8)$ & 16 & $(24,5)$ & 18 & \\
\hline Etapa II & 10 & $(24,4)$ & 32 & $(49,2)$ & 42 & \\
\hline Etapa III & 22 & $(53,7)$ & 11 & $(16,9)$ & 33 & $<0,01$ \\
\hline Etapa IV & 7 & (17) & 6 & $(9,2)$ & 13 & \\
\hline
\end{tabular}

XRT - QMT: Quimioradioterapia.

del mesorrecto enteramente satisfactoria, con margen circunferencial y distal libres de tumor y ausencia de metástasis linfáticas, desarrollan una recidiva local o metástasis a distancia. Ante esta situación la primera reacción es buscar algún factor no reconocido que sea la causa de esta recidiva tumoral y su curso desfavorable. Un hallazgo relativamente frecuente es la presencia de NTM y pudieran ser estos depósitos un marcador de agresividad del tumor que merece nuestra atención. Es difícil dar una cifra de la incidencia de los NTM por varias razones. Los escasos estudios publicados son retrospectivos, las técnicas de estudio de las piezas operatorias en Anatomía Patológica son diferentes y el reconocimiento de los NTM como un depósito tumoral distinto de las metástasis linfáticas no es uniforme. En consecuencia, es claro que por estas razones el conocimiento y significado pronóstico de estos nódulos es todavía incierto lo que genera sistematizaciones o clasificaciones que en relación a la AJCC tendrán un valor temporal mientras no hayan estudios más extensos y prospectivos.

Por lo expuesto anteriormente, la frecuencia con la que se presentan NTM puede ser muy variable. En estudios realizados por Ono y cols. y Ratto y cols. ${ }^{4,5}$ se encontraron en el $7,5 \%$ y $14 \%$, respectivamente, de las piezas operatorias de pacientes operados por un cáncer de recto. En nuestra serie los NTM se encontraron en el $16 \%$ de las piezas operatorias. Incluso fueron detectadas en 12 de 31 pacientes $(38 \%)$ sometidos a XRT-QMT preoperatoria. En este estudio además se encontró que la presencia de NTM se relacionaba significativamen- te con parámetros como: menor grado de diferenciación histológica; presencia de permeaciones linfáticas y perineurales; metástasis ganglionares linfáticas y etapa tumoral avanzada.

Sería de particular importancia determinar si la presencia de NTM en pacientes sin metástasis ganglionares determina un pronóstico similar al de los pacientes con ellas. En nuestro estudio 15 de 41 $(36,6 \%)$ pacientes tenían NTM no asociado a metástasis linfáticas en el mesorrecto. En la serie de Prabhudesai y cols. ${ }^{6}, 7$ de 29 pacientes (24\%) tenían NTM sin metástasis linfáticas asociadas. El escaso número de pacientes en este sub-grupo impide conclusiones valederas en términos de sobrevida, pero a pesar de este hecho, la presencia de NTM en pacientes sin metástasis linfáticas, se le considera, con fines prácticos, como etapa III $\left(\mathrm{N}_{+}\right)$, particularmente en la indicación de tratamientos complementarios a la cirugía. En esta misma comunicación ${ }^{6}$ se demostró la asociación entre la presencia de NTM y permeaciones vasculares, linfáticas y perineurales y también se encontró que no hubo diferencia significativa en el número de NTM entre los pacientes que recibieron XRT-QMT y aquellos que no fueron sometidos a esta modalidad de tratamiento. Esto último, es concordante con la serie de Ratto y cols ${ }^{5}$ y también lo comprobamos en nuestro estudio.

En relación al pronóstico, en esta serie, no se demostró que la presencia de NTM afecte significativamente las cifras de sobrevida actuarial a 5 y 10 años, sin embargo, se observa una tendencia a una menor sobrevida de los pacientes con NTM, que 
posiblemente no es estadísticamente demostrada dado el tamaño de la muestra. Estos hallazgos son enteramente similares a los observados en el estudio de Prabhudesai y cols., quienes además, tampoco encontraron diferencias significativas en la sobrevida actuarial en un plazo menor (3 años). En este último estudio sí demostró que las metástasis a distancia se desarrollan más precozmente en los pacientes con NTM.

La patogenia o el origen de los NTM es todavía poco clara. Se tiende a aceptar que representan ganglios linfáticos reemplazados totalmente por tejido tumoral metastásico, sin embargo, una proporción importante de los NTM son microscópicos, por lo tanto, menores que el tamaño de un ganglio linfático. Estos hallazgos hacen a algunos plantear que tendrían mayor impacto pronóstico negativo la presencia de NTM microscópicos ó menores que 3 mms porque representarían una extensión discontinua del cáncer del recto por vía, posiblemente hematógena, expresando así una mayor agresividad del tumor primitivo En una publicación reciente de Ueno y cols. ${ }^{7}$ sobre 427 enfermos sometidos a resección por cáncer del recto, se demostraron NTM microscópicos ( $\leq 1 \mathrm{~mm})$ en $96(22,5 \%)$. La incidencia de NTM microscópicos fue significativamente mayor en tumores menos diferenciados; con extensa invasión de la pared: con infiltración linfática y venosa en el tumor primario y con metástasis linfáticas. Además, los pacientes con NTM microscópicos mostraron una significativa menor sobrevida y la recurrencia local fue significativamente mayor que en pacientes sin NTM.
En conclusión: la presencia de NTM se relaciona con una etapa más avanzada y con factores anatomopatológicos de mal pronóstico, sin embargo, según este estudio no afectarían la recurrencia, ni la sobrevida libre de enfermedad.

\section{REFERENCIAS}

1. American Joint Committee on Cancer Manual. for staging of cancer. Philadelphia. Lippincott- Raven, 1998; 83-88.

2. Royal College of Pathologists. Standards and Minimum Datasets for Reporting Common Cancers: Minimum dataset for colorectal cancer histopathology reports. July 1998.

3. Scott N, Jackson P, Jaberi AL, Dixon MF, Quirke $P$, and Finan PJ. Total mesorectal excision and local recurrence: a study of tumour spread in the mesorectum distal to rectal cancer. Br J Surg 1995; 82: 1031-1033.

4. Ono C, Yoshinagan K. Discontinuous rectal cancer spread in the mesorectum and the optimal distal clearence margin in situ. Dis Colon Rectum 2002; 45: 744-749.

5. Ratto C, Ricci R, Rossi C, Morelli U, Vecchio FM, Doglietto GB. Mesorectal microfoci adversely affect the prognosis of patients with rectal cancer. Dis colon rectum 2002; 45: 733-743.

6. Prabhudesai A, Arif S, Finlayson C, Kumar D. Impact of Microscopic Extranodal Tumor Deposits on the Outcome of Patients With Rectal Cancer. Dis Colon Rectum 2003; 46: 1531-1537.

7. Ueno $H$, Muchizuki $H$, Tamakuma S. Prognostic significance of extranodal microscopic foci discontinuous with primary lesion in rectal cancer. Dis Colon Rectum 1998; 41: 55-61 